\title{
Direct mapping of the temperature and velocity gradients in discs Imaging the vertical $\mathrm{CO}$ snow line around IM Lupi
}

\author{
C. Pinte ${ }^{1,2,3}$, F. Ménard ${ }^{1}$, G. Duchêne $e^{4,1}$, T. Hill ${ }^{5}$, W. R. F. Dent ${ }^{5}$, P. Woitke ${ }^{6}$, S. Maret ${ }^{1}$, G. van der Plas ${ }^{1}$, \\ A. Hales ${ }^{5,7}$, I. Kamp ${ }^{8}$, W. F. Thi ${ }^{9}$, I. de Gregorio-Monsalvo ${ }^{5}$, C. Rab ${ }^{8}$, S. P. Quanz ${ }^{10}$, H. Avenhaus ${ }^{10,11}$, \\ A. Carmona ${ }^{12}$, and S. Casassus ${ }^{11}$ \\ 1 Univ. Grenoble Alpes, CNRS, IPAG, 38000 Grenoble, France \\ e-mail: christophe.pinte@univ-grenoble-alpes.fr \\ 2 UMI-FCA, CNRS/INSU, France (UMI 3386), and Dept. de Astronomía, Universidad de Chile, Santiago, Chile \\ 3 Monash Centre for Astrophysics (MoCA) and School of Physics and Astronomy, Monash University, Clayton Vic 3800, Australia \\ 4 Astronomy Department, University of California, Berkeley, CA 94720-3411, USA \\ 5 Atacama Large Millimeter / Submillimeter Array, Joint ALMA Observatory, Alonso de Córdova 3107, Vitacura 763-0355, \\ Santiago, Chile \\ ${ }^{6}$ Centre for Exoplanet Science, SUPA, School of Physics and Astronomy, University of St Andrews, North Haugh, St Andrews, \\ Fife, KY16 9SS, UK \\ 7 National Radio Astronomy Observatory, 520 Edgemont Road, Charlottesville, Virginia 22903-2475, USA \\ 8 Kapteyn Astronomical Institute, University of Groningen, Postbus 800, 9700 AV Groningen, The Netherlands \\ 9 Max Planck Institute for Extraterrestrial Physics, Giessenbachstrasse, 85741 Garching, Germany \\ ${ }^{10}$ Institute for Astronomy, ETH Zurich, Wolfgang-Pauli-Strasse 27, 8093 Zurich, Switzerland \\ 11 Departamento de Astronomiía, Universidad de Chile, Casilla 36-D, Santiago, Chile \\ 12 IRAP, Université de Toulouse, CNRS, UPS, 31400 Toulouse, France
}

Received 15 June 2017 / Accepted 28 September 2017

\begin{abstract}
Accurate measurements of the physical structure of protoplanetary discs are critical inputs for planet formation models. These constraints are traditionally established via complex modelling of continuum and line observations. Instead, we present an empirical framework to locate the CO isotopologue emitting surfaces from high spectral and spatial resolution ALMA observations. We apply this framework to the disc surrounding IM Lupi, where we report the first direct, i.e. model independent, measurements of the radial and vertical gradients of temperature and velocity in a protoplanetary disc. The measured disc structure is consistent with an irradiated self-similar disc structure, where the temperature increases and the velocity decreases towards the disc surface. We also directly map the vertical CO snow line, which is located at about one gas scale height at radii between 150 and 300 au, with a CO freeze-out temperature of $21 \pm 2 \mathrm{~K}$. In the outer disc $(>300 \mathrm{au})$, where the gas surface density transitions from a power law to an exponential taper, the velocity rotation field becomes significantly sub-Keplerian, in agreement with the expected steeper pressure gradient. The sub-Keplerian velocities should result in a very efficient inward migration of large dust grains, explaining the lack of millimetre continuum emission outside of $300 \mathrm{au}$. The sub-Keplerian motions may also be the signature of the base of an externally irradiated photo-evaporative wind. In the same outer region, the measured $\mathrm{CO}$ temperature above the snow line decreases to $\approx 15 \mathrm{~K}$ because of the reduced gas density, which can result in a lower $\mathrm{CO}$ freeze-out temperature, photo-desorption, or deviations from local thermodynamic equilibrium.
\end{abstract}

Key words. protoplanetary disks - circumstellar matter - accretion, accretion disks - radiative transfer - stars: formation stars: individual: IM Lupi

\section{Introduction}

The initial stages of planet formation are the results of a complex interplay between several physical mechanisms which all depend on the disc density, temperature, chemical abundances, and velocity structure (Testi et al. 2014). A critical step is the growth of submicron-sized grains, originating from the parent cloud, into large planetesimals within the few million years of a protoplanetary disc lifetime. Dust grains are believed to grow by collision and sticking (Blum \& Wurm 2008; Dominik et al. 2007). In parallel, they settle towards the disc midplane and migrate inwards as a result of the conjugate actions of stellar gravity, aerodynamical gas drag, and radial pressure gradient (e.g. Weidenschilling 1977). The radial concentration of dust grains depends strongly on the sub-Keplerian gas velocity, pressure gradients (e.g. Pinilla et al. 2012), and disc thermal structure (Laibe et al. 2012; Pinte \& Laibe 2014). In particular, molecular snow lines - the two-dimensional surface where abundant volatiles condensate onto dust grains - result in increased solid particle stickiness and dust-to-gas ratio, promoting particle growth (e.g. Ciesla \& Cuzzi 2006; Ros \& Johansen 2013).

These dynamical processes remain hard to constrain by existing observations, however. Rotational molecular lines are a powerful tool that can be used to probe the disc structure, but constraints rely in most cases on complex model fitting or on indirect methods. For instance, detailed radiative transfer 
Table 1. Summary of observations.

\begin{tabular}{lccc}
\hline \hline Wavelength or line & Disc-integrated flux & Beam & Image or moment 0 rms $(1 \sigma)$ \\
\hline $1.3 \mathrm{~mm}$ cont. & $225 \pm 23 \mathrm{mJy}^{-1}$ & $0.36^{\prime \prime} \times 0.55^{\prime \prime}$ at $-80^{\circ}$ & $0.05 \mathrm{mJy} / \mathrm{beam}$ \\
${ }^{12} \mathrm{CO}(2-1)$ & $24.1 \pm 2.4 \mathrm{Jy} \mathrm{km} \mathrm{s}^{-1}$ & $0.36^{\prime \prime} \times 0.56^{\prime \prime}$ at $-78^{\circ}$ & $3.5 \mathrm{mJy} \mathrm{km} \mathrm{s}^{-1}$ \\
${ }^{13} \mathrm{CO}(2-1)$ & $7.9 \pm 0.8 \mathrm{Jy} \mathrm{km} \mathrm{s}^{-1}$ & $0.38^{\prime \prime} \times 0.58^{\prime \prime}$ at $-79^{\circ}$ & $4.2 \mathrm{mJy} \mathrm{km} \mathrm{s}^{-1}$ \\
$\mathrm{C}^{18} \mathrm{O}(2-1)$ & $1.3 \pm 0.2 \mathrm{Jy} \mathrm{km} \mathrm{s}^{-1}$ & $0.38^{\prime \prime} \times 0.58^{\prime \prime}$ at $-78^{\circ}$ & $3.9 \mathrm{mJy} \mathrm{km} \mathrm{s}^{-1}$ \\
\hline
\end{tabular}

Notes. The continuum flux was measured over the area where the signal is greater than 3 times the map rms (estimated far from the source). The line flux was measured by integrating the flux where the signal is greater than 3 times the per channel RMS, estimated from the scatter in line-free channels.

modelling of multiple CO lines can provide an estimate of the vertical temperature structure of the disc (Dartois et al. 2003), indicate freeze-out of the gas phase molecules onto dust grains in the cold midplane (e.g. Qi et al. 2011; Zhang et al. 2017), and suggest a very low level of turbulence (e.g. Flaherty et al. 2015). Indirect mapping via chemical imaging (Qi et al. 2013, with $\mathrm{N}_{2} \mathrm{H}^{+}$; and Mathews et al. 2013, with $\mathrm{DCO}^{+}$) has also been used to estimate the location of the $\mathrm{CO}$ snow line. However, Aikawa et al. (2015), van't Hoff et al. (2017), and Huang et al. (2017) have shown that these species are not robust CO snow line tracers, and that detailed chemical modelling is required to infer the midplane $\mathrm{CO}$ snow line location from $\mathrm{N}_{2} \mathrm{H}^{+}$or $\mathrm{DCO}^{+}$observations.

The combination of high spatial and spectral resolution and sensitivity offered by ALMA opens new avenues to directly map the disc thermal and kinematic structure by resolving the gas disc both radially and vertically. Dutrey et al. (2017) recently introduced a method to map the thermal and density gas structure of discs at close to edge-on inclinations. Discs at intermediate inclinations are also ideal targets as the Keplerian velocities spatially separate the emitting regions, eliminating line of sight confusion (e.g. de Gregorio-Monsalvo et al. 2013; Rosenfeld et al. 2013).

IM Lupi is a M0V T Tauri star (distance of $161 \pm 10 \mathrm{pc}$, Gaia Collaboration 2016) surrounded by a large and massive disc with an inclination of $48^{\circ} \pm 3^{\circ}$ (Pinte et al. 2008; Cleeves et al. 2016). The disc is detected in rotational CO emission up to a radius of $970 \mathrm{au}$, in the millimetre continuum up to 310 au (Panić et al. 2009; Cleeves et al. 2016), and in scattered light up to 320 au with a well-defined disc morphology (Pinte et al. 2008, rescaled to the updated distance), but with an extended component up to 720 au in radius. We present here a framework to directly measure the altitude, velocity, and temperature of the CO layers from new high spectral $\left(0.05 \mathrm{~km} \mathrm{~s}^{-1}\right)$ and intermediate spatial $\left(0.4^{\prime \prime}\right)$ resolution ALMA observations of the disc surrounding IM Lupi.

\section{Observations and data reduction}

IM Lupi was observed with ALMA in band 6 on the night from 9 to 10 June 2015 with a total on-source time of $37.4 \mathrm{~min}$ (Program ID 2013.1.00798.S). The array was configured with 37 antennas and baselines ranging from 21.4 to $784 \mathrm{~m}$. Titan was used as a flux calibrator and the quasars J1517-2422 and J1610-3958 were used as bandpass and phase calibrators. Two of the four spectral windows of the correlator were configured in time division mode (TDM) centred at $218.9 \mathrm{GHz}$ and $231.7 \mathrm{GHz}$, each with $1.875 \mathrm{GHz}$ usable bandwidth. The other two spectral windows were configured in frequency division mode (FDM) to target the ${ }^{12} \mathrm{CO} J=2-1,{ }^{13} \mathrm{CO} J=2-1$, and $\mathrm{C}^{18} \mathrm{O} J=2-1$ transitions, with a channel spacing of $30.5 \mathrm{kHz}$ and a channel averaging of 2. Because of the Hanning smoothing used to reduce spectral ringing, the actual spectral resolution is $35 \mathrm{kHz}$, corresponding to $45-48 \mathrm{~m} / \mathrm{s}$ depending on the line. The median partial water vapour was $0.93 \mathrm{~mm}$. The data sets were calibrated using the Common Astronomy Software Applications pipeline (CASA, McMullin et al. 2007, version 4.6.0). We performed three successive rounds of phase self-calibration, the last with solutions calculated for each individual integration (6s), followed by a phase and amplitude self-calibration. The continuum self-calibration solutions were applied to the CO lines. A CLEAN mask was manually defined for the continuum image and each channel map. Channel maps were produced both with and without continuum subtraction (using the CASA task uvcon$t s u b)$. We estimate the flux calibration uncertainty to $10 \%$.

The disc is spatially resolved in all transitions and channel maps show the typical butterfly pattern of discs in Keplerian rotation. The measured fluxes are presented in Table 1 and the representative channel maps for ${ }^{12} \mathrm{CO}$ and ${ }^{13} \mathrm{CO}$ in Fig. 1. The $\mathrm{C}^{18} \mathrm{O}$ channel maps and continuum image are shown in Figs. A.1 and C.1, respectively. Our data and results are consistent with those of Cleeves et al. (2016). We focus in the following on the spatial distribution of the emission in individual channel maps.

\section{Reconstructing the altitude, velocity, and temperature of the $\mathrm{CO}$ emitting layers}

In a given channel map, the line emission is concentrated along the isovelocity curve, i.e. the set of points where the projected velocity of the emitting surface is constant. As a result, the emission originating from the upper and lower surfaces (i.e. above and below the midplane as seen from the observer), and from the near and far sides of these surfaces, is well separated (Figs. 2 and 1). By locating the emission in each channel map, we can directly reconstruct, via simple geometrical arguments, the position and velocity of each of the CO layers.

\subsection{Altitude}

We consider the coordinate system where the $x$-axis is aligned with the disc major axis, and we denote $\left(x_{\star}, y_{\star}\right)$ the position of the star which was determined by the peak of the continuum map (Fig. 2). Because the gas is vertically pressure supported, we assume that, at any point in the disc, the gas is rotating on a circular orbit parallel to the disc midplane, i.e. we assume that the bulk of the motion is described by Keplerian rotation and neglect for this analysis any radial or vertical gas circulation. The radius and altitude of this orbit are denoted by $r$ and $h$, respectively.

For a given offset $\delta x=x-x_{\star}$ in the image plane, a vertical line will intersect the isovelocity curve of the upper disc 
C. Pinte et al.: Direct mapping of the temperature and velocity gradients in discs
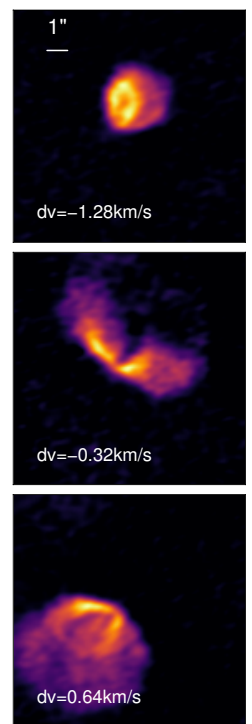
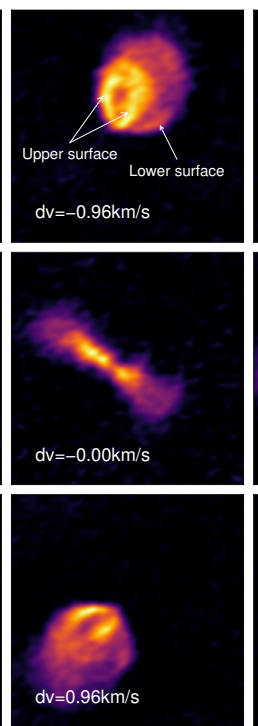
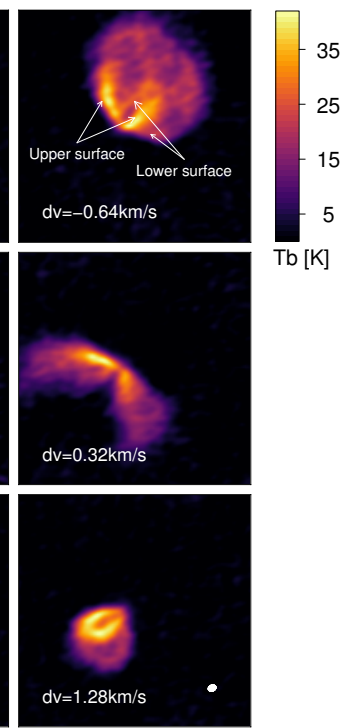
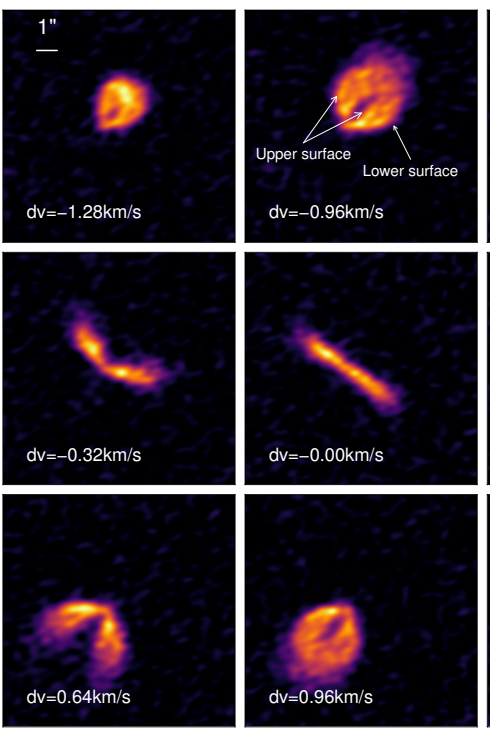
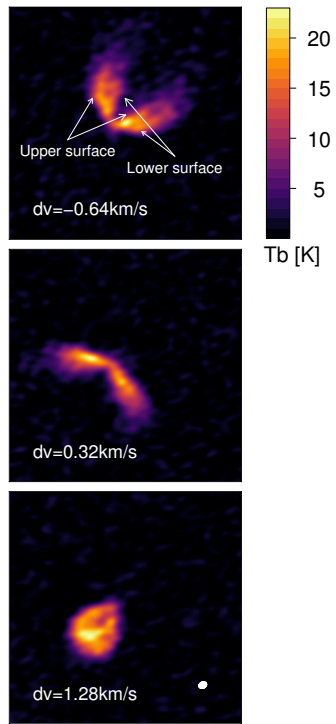

Fig. 1. Channel maps of ${ }^{12} \mathrm{CO}($ left $)$ and ${ }^{13} \mathrm{CO}$ (right) displayed with a channel width of $80 \mathrm{~m} / \mathrm{s}$. The data is not continuum subtracted. The d $v$ is relative to the systemic velocity of $4.5 \mathrm{~km} \mathrm{~s}^{-1}$. The beam is indicated in the bottom right corner.

surface $^{1}$ at two points (for small enough $x$ ), which belong to the same orbit, i.e. at the same distance from the star and at the same altitude. Due to line broadening, and to finite spatial and spectral resolution, the emission is not located exactly on the isovelocity curve, but forms a narrow band around it. We estimate the position of the emission by its maximum, as illustrated in Fig. 2. If we denote $y_{\mathrm{n}}$ and $y_{\mathrm{f}}$ the ordinates of the two points on the near and far side of the disc, the coordinates of the centre of the projected circular orbit passing through those two points are $\left(x_{\mathrm{c}}, y_{\mathrm{c}}\right)$, where $x_{\mathrm{c}}=x_{\star}$ and $y_{\mathrm{c}}=\left(y_{\mathrm{f}}+y_{\mathrm{n}}\right) / 2$. Any point on this ellipse fulfils $\left(x-x_{\star}\right)^{2}+\left(\left(y-y_{\mathrm{c}}\right) / \cos i\right)^{2}=r^{2}$, where the orbital radius $r$ is the length of the semi-major axis of the ellipse and $i$ the disc inclination.

By using the point on the far side of the disc surface, we can for instance derive

$$
r=\sqrt{\left(x-x_{\star}\right)^{2}+\left(\frac{y_{\mathrm{f}}-y_{\mathrm{c}}}{\cos i}\right)^{2}} .
$$

The altitude $h$ of the orbit is derived by noting that the offset between the centre of the ellipse and the star is simply $h \sin i$ :

$h=\frac{y_{\mathrm{c}}-y_{\star}}{\sin i}$

\subsection{Gas velocity}

In a given velocity channel, the projected radial velocity $v_{\text {obs }}$ is known and can be expressed as $v_{\text {obs }}=v_{\text {syst }}+v \cos \theta \sin i$, where $v$ is the actual gas velocity around the central star, $v_{\text {syst }}$ the systemic velocity, and $\theta$ is the true longitude. By noting that $\cos \theta=\left(x-x_{\star}\right) / r$, we obtain the actual gas velocity at each point:

$v=\left(v_{\mathrm{obs}}-v_{\mathrm{syst}}\right) \frac{r}{\left(x-x_{\star}\right) \sin i}$.

\footnotetext{
1 The same formalism can be used for the lower surface, but for reasons we detail in Sect. 4, it cannot be used here and we only describe the formalism for the upper surface.
}

\subsection{Gas kinetic temperature}

For optically thick emission, and as long as the emission fills the beam, the observed brightness temperature $T_{\mathrm{b}}$ is very close to the excitation temperature $T_{\mathrm{ex}}, T_{\mathrm{b}}=T_{\mathrm{ex}}(1-\exp (-\tau)$ ). Low $J$ CO lines are close to local thermodynamic equilibrium, with an excitation almost equal to the gas kinetic temperature $T_{\text {kin }}$ (e.g. Pavlyuchenkov et al. 2007). For optically thick lines, the peak intensity then provides an accurate estimate of the gas temperature. This is the case for the ${ }^{12} \mathrm{CO}$ and ${ }^{13} \mathrm{CO}$ lines (see discussion in Sect. 4), but the $\mathrm{C}^{18} \mathrm{O}$ emission is partly optically thin and the brightness temperature is lower than the actual excitation temperature. To reconstruct the disc temperature profile, we measured the brightness temperature at all points extracted above. We also extracted the brightness temperature of points on the disc lower surface in the same way (Fig. 2).

\subsection{Reconstructing the full spatial, kinematic, and thermal structure of the CO layers}

As described above, by locating the position of the maxima in each isovelocity curve and for each $x$ in a given channel map (where we sampled $x$ every $0.08^{\prime \prime}$, i.e. $\approx 1 / 4$ of the beam), we can directly measure $r$ and the corresponding elevation $h$ and velocity $v$, and we can estimate $T_{\text {gas }}$.

Each channel probes a range of distances from the star depending on the velocity. By repeating this procedure for each channel, we can reconstruct the complete $2 \mathrm{D}$ velocity and temperature distribution of the $\mathrm{CO}$ emitting layers, as shown in Fig. 4.

\subsection{Caveats}

The central channels of ${ }^{12} \mathrm{CO}$ (within $\pm 0.7 \mathrm{~km} \mathrm{~s}^{-1}$ from the systemic velocity) suffer from strong foreground extinction (Panić et al. 2009; Cleeves et al. 2016). They were excluded from the analysis for the temperature estimate. The morphology of the channel maps is not affected, however, and we included them to measure the altitude and velocity.

The method presented in this section can only be applied in regions where the emission originates from a vertically thin 
layer, so that an altitude $h$ can be defined, i.e. a clear maximum can be detected in the channel map. This is only possible when the projected width of the emitting layer is significantly smaller than the beam. This is the case for instance when the molecular layer itself is a narrow layer, or when the optically depth gradient is steep around the $\tau=1$ surface and we can only see a narrow layer.

These conditions are met for the $\mathrm{CO}$ layers of IM Lupi up to a radius $450 \mathrm{au}$, where we are able to distinctly identify the location of the isovelocity curves, but are no longer valid far from the star even though the emission is detected up to $\approx 1000 \mathrm{au}$. As already noted by Cleeves et al. (2016), the CO emission at large scales ( $>450 \mathrm{au}$ ) becomes very diffuse, making it impossible to locate the isovelocity curve and to estimate an altitude. This is very likely the result of UV photo-desorption of the CO by external irradiation from the interstellar radiation field (see e.g. Fig. 5d), allowing gas $\mathrm{CO}$ to exist even in the midplane and to emit from a vertically extended region.

The presented framework also requires sufficient signal in a given channel to accurately locate the emission. For ${ }^{12} \mathrm{CO}$ we were able to use the intrinsic channel width, but for ${ }^{13} \mathrm{CO}$ and $\mathrm{C}^{18} \mathrm{O}$ we needed to bin the data to channel widths of 80 and $320 \mathrm{~m} / \mathrm{s}$, respectively. This introduces spatial smearing due to the disc Keplerian velocity and reduces correspondingly the range over which we can accurately measure the $\mathrm{CO}$ layer altitude ${ }^{2}$. For ${ }^{13} \mathrm{CO}$ and $\mathrm{C}^{18} \mathrm{O}$, this prevented a measurement of the altitude at radii smaller than $\approx 150$ au and $\approx 220$ au, respectively.

Continuum subtraction can significantly affect the measured brightness temperature and apparent morphology of line emission when the line is optically thick, and the line and continuum intensities are comparable (Boehler et al. 2017). At the centre of an optically thick line, the dust continuum is absorbed by the molecule. As the continuum is usually estimated from linefree channels, this results in oversubtraction of the continuum close to the line centre. The authors conclude that using noncontinuum-subtracted maps is preferable in order to estimate the line brightness temperature (see e.g. their Fig. 8). This conclusion also holds for the framework we have presented, and using non-continuum-subtracted channel maps will provide a more accurate estimate of the brightness temperature and will prevent artificial spatial shift of the line emission due to oversubtraction of the local continuum emission.

At the spatial resolution of our observations, the continuum subtraction has no impact on the results as the continuum is optically thin in the regions where we can isolate the various isovelocity curves, and the corresponding continuum intensity is negligible compared to the line intensity. As a sanity check, we performed our analysis on both the continuum-subtracted and non-subtracted maps and did not find any significant difference. At higher spatial resolution, however, the effect is very likely to become important as the continuum emission in our current central beam is significant, producing a central "hole" in the continuum-subtracted ${ }^{13} \mathrm{CO}$ and $\mathrm{C}^{18} \mathrm{O}$ maps (Öberg et al. 2015; Cleeves et al. 2016), which is absent in the non-continuumsubtracted maps (Figs. 1 and A.1).

\footnotetext{
2 This mainly affects our ability to measure $h$, which depends on the small distance between the star's position and the centre of the gas orbit. The radius and velocity are less affected as they depend on greater lengths in the channel map.
}

\section{Results and discussion}

\subsection{CO layers}

As expected, we find that the ${ }^{12} \mathrm{CO}$ emitting layer lies higher in the disc than the ${ }^{13} \mathrm{CO}$, due to its much higher optical depth (Fig. 4, left panel). This is readily visible in Fig. 1, where the two surfaces are geometrically much closer to one another for ${ }^{13} \mathrm{CO}$ than ${ }^{12} \mathrm{CO}$. While already suggested by previous ALMA observations (e.g. de Gregorio-Monsalvo et al. 2013; Rosenfeld et al. 2013, for HD 163296), this is the first direct, model-independent measurement of the altitude of the $\mathrm{CO}$ layers. In particular, we clearly see that $\mathrm{CO}$ surfaces are flaring and seem to flatten out outside of $\approx 300 \mathrm{au}$. At a distance of $200 \mathrm{au}$, the ${ }^{12} \mathrm{CO}$ emission originates from an altitude of $\approx 65 \mathrm{au}$, while the ${ }^{13} \mathrm{CO}$ originates from $\approx 25 \mathrm{au}$. This corresponds to about 2.5 and 1 hydrostatic scale height, respectively. Interestingly, these values are very similar to the numbers that Dartois et al. (2003) and Dutrey et al. (2017) have found for DM Tau and the Flying Saucer, respectively.

A linear regression analysis indicates a power-law exponent of $1.8 \pm 0.2$ and $2.1 \pm 0.4$ for the altitude of ${ }^{12} \mathrm{CO}$ and ${ }^{13} \mathrm{CO}$ layers at radii smaller than $300 \mathrm{au}$, respectively. It should be noted that these values are much larger than the actual faring index of the disc pressure scale height $(\approx 1.15-1.2$ in the various published disc models). This is expected, however, as the shape of the emitting $\mathrm{CO}$ layer is set by the irradiation from the star (responsible for both the photo-dissociation and disc temperature). The emitting molecular layer should roughly follow a layer with a constant optical depth as seen from the star, and not the actual disc scale height. Similarly, large exponents were measured around the Herbig star HD 97048 for the PAH emission (Lagage et al. 2006) and scattered light (Ginski et al. 2016) layers, which are also set by the stellar irradiation.

The $\mathrm{C}^{18} \mathrm{O}$ emission is more difficult to locate due to the lower signal-to-noise ratio and required velocity binning, but it appears co-spatial with the ${ }^{13} \mathrm{CO}$ emission. Since ${ }^{13} \mathrm{CO}$ and $\mathrm{C}^{18} \mathrm{O}$ have a typical optical depth ratio of about $8: 1$, one expects the $\mathrm{C}^{18} \mathrm{O}$ to be closer to the midplane than ${ }^{13} \mathrm{CO}$ by the same reasoning as for ${ }^{13} \mathrm{CO}$ and ${ }^{12} \mathrm{CO}$. The fact that we do not see any significant difference in altitude between ${ }^{13} \mathrm{CO}$ and $\mathrm{C}^{18} \mathrm{O}$ suggests that we are actually probing a physical layer in the disc below which there is no significant $\mathrm{CO}$ emission, hence this layer marks the vertical freeze-out line.

\subsection{CO depleted midplane}

The disc lower surface (i.e. on the other side of the disc midplane) is clearly visible, in particular for ${ }^{12} \mathrm{CO}$, telling us that the $\mathrm{CO}$ gas abundance is significantly reduced in the disc midplane. To illustrate this, we generated a representative disc model (Fig. 5) using the radiative transfer code MCFOST (Pinte et al. 2006, 2009). This model qualitatively explains the observed morphological features, but is not in any sense optimized and should only be considered for general guidance. We also produced a few additional models where we modified physical ingredients (UV chemistry, $\mathrm{CO}$ condensation) to discuss their impact on the observed channel maps (Fig. 5). The model description and parameters are presented in Appendix B. In particular, we see that the $\mathrm{CO}$ needs to be physically depleted in the disc midplane to be able to observe the lower disc surface. The temperature gradient alone (Fig. 5, panel a) results in a strong attenuation by the cold $\mathrm{CO}$ in the midplane, masking the lower surface. 


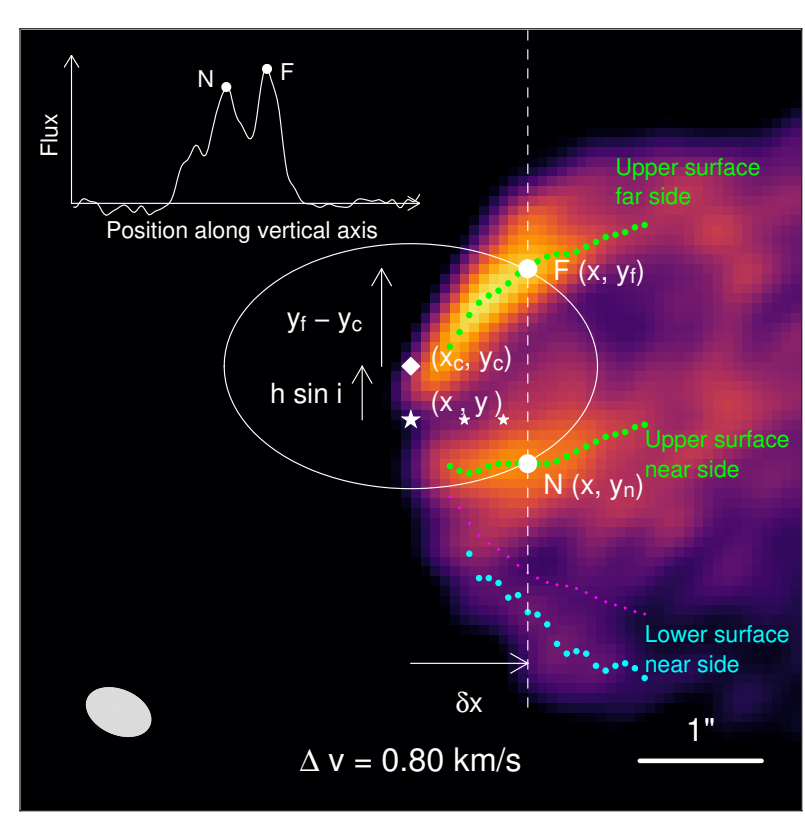

Fig. 2. Single ${ }^{12} \mathrm{CO}$ channel with a schematic of the quantities we measure. The disc has been rotated so that the semi-major axis is horizontal. The position of the central object is marked by a star. For a given offset $\delta x$ along the disc major axis, two maxima $\mathrm{N}$ and $\mathrm{F}$ on the same vertical are located on the near and far sides of the disc's upper surface. These two points are on the same inclined circular orbit (white ellipse), whose centre is marked by a diamond. Indicated are the geometrical parameters from which the cylindrical radius, height, and velocity of the points $\mathrm{F}$ and $\mathrm{B}$ can be measured. By repeating the procedure for each $\delta x$ (green points, sampled every $0.08^{\prime \prime}$ ) and each velocity channel, a full mapping of the $\mathrm{CO}$ upper surface is performed. The cyan points mark the location of the lower surface (near side), while the magenta points represent the symmetric of the far upper surface relative to the disc major axis.

Because the ${ }^{12} \mathrm{CO}$ emission on the lower surface is seen through the disc, it should originate from the lowest boundary of the actual CO layer, i.e. exactly at the vertical CO snow line. It can potentially provide a more accurate estimate of the altitude of the CO snow line, and of the CO layer thickness by comparison with measurements from the upper surface. We attempted to locate the ${ }^{12} \mathrm{CO}$ lower surface using the same formalism as for the upper surface. But, surprisingly, the lower surface appears shifted further away from the midplane than the upper surface is (Fig. 2), indicating an apparent higher altitude, while one would expect the opposite (see Fig. 3). The ${ }^{13} \mathrm{CO}$ does not show any shift on the other hand. The absence of shift is consistent with the ${ }^{13} \mathrm{CO}$ emission probing the actual physical layer of $\mathrm{CO}$. We interpret the apparent shift of the lower ${ }^{12} \mathrm{CO}$ surface as a radiative transfer effect.

If the $\mathrm{CO}$ is entirely depleted at temperatures below $20 \mathrm{~K}$, the lower disc surface appears very wide and uniformly bright in the model, with no shift relative to the upper surface (Fig. 5b). To reproduce the observed morphology, we need a partial depletion of $\approx 10^{-4}$ (i.e. an abundance of $\approx 10^{-8}$ ) of $\mathrm{CO}$ where $T<20 \mathrm{~K}$ (Fig. 5c). The exact value is not well constrained and depends on other parameters, in particular the local turbulent velocity dispersion. This small fraction of cold CO gas below the snow line attenuates the centre of the emission from the lower surface. Emission can only escape where the velocity shift is large enough, i.e. slightly away from the isovelocity curve, resulting in an apparent spatial shift of the lower surface. In our simple model, this is reproduced by a constant depletion factor. In reality, this means

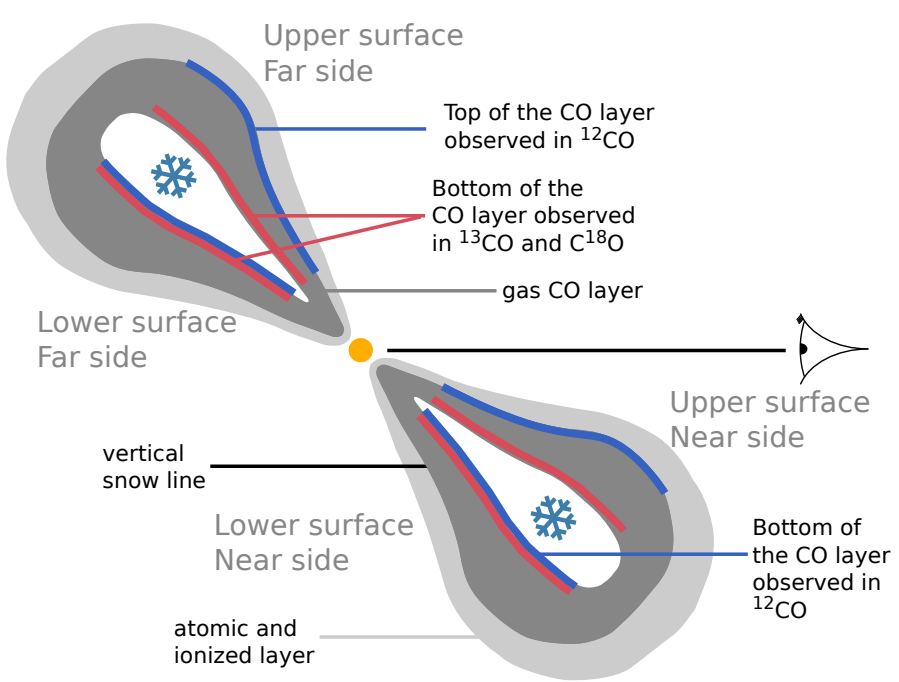

Fig. 3. Schematic view of the various layers observed in the $\mathrm{CO}$ lines.

that the vertical snow line is not infinitely sharp, as expected, but with a $\mathrm{CO}$ abundance that decreases progressively with altitude, i.e. over a few K. It is potentially a powerful diagnostic to constrain the physics of the $\mathrm{CO}$ condensation on grains, and the possible vertical "smearing" of the ice line due to turbulent vertical mixing (Aikawa 2007; Furuya \& Aikawa 2014).

\subsection{Vertical velocity gradient and sub-Keplerian rotation}

The different altitudes probed by the $\mathrm{CO}$ isotopologues also allow for the first measurement of the vertical gradient on the rotational velocity of the disc. At high altitude, the ${ }^{12} \mathrm{CO}$ emitting layer is rotating slower than the ${ }^{13} \mathrm{CO} / \mathrm{C}^{18} \mathrm{O}$ emitting layer, which is located closer to the midplane (Fig. 4, central panel).

Within $300 \mathrm{au}$, the difference in the measured azimuthal velocities between ${ }^{12} \mathrm{CO}$ and ${ }^{13} \mathrm{CO} / \mathrm{C}^{18} \mathrm{O}$ is in good agreement with the vertical dependence of the stellar gravitational field (Fig. 4), where the radial projection of the gravitational force per unit mass decreases with altitude:

$\frac{v_{\mathrm{Kep}}^{2}}{r}=\frac{r}{\sqrt{r^{2}+h^{2}}} \times \frac{G M_{\star}}{r^{2}+h^{2}}$.

At larger radii, the gas becomes clearly sub-Keplerian. This can be understood by including the pressure gradient term (e.g. Rosenfeld et al. 2013):

$\frac{v^{2}}{r}=\frac{G M_{\star} r}{\left(r^{2}+h^{2}\right)^{3 / 2}}+\frac{1}{\rho_{\mathrm{gas}}} \frac{\partial P}{\partial r}$.

With both the density and temperature profile generally decreasing with distance, this term is negative, reducing the gas velocity compared to the fiducial Keplerian velocity. This phenomenon is thought to play an important role in the radial migration and growth of solids in these discs (Weidenschilling 1977; Barrière-Fouchet et al. 2005; Brauer et al. 2008).

Interestingly, the radius at which we see significant subKeplerian velocities coincides with the radius where the gas surface density transitions from a power law to a tapered profile (Panić et al. 2009; Cleeves et al. 2016), i.e. where the disc pressure gradient is becoming larger and where the velocities should deviate the most from Keplerian. At a radius of $400 \mathrm{au}$, the measured deviations from the altitude dependent Keplerian rotation are of the order of $0.15 \mathrm{~km} \mathrm{~s}^{-1}$ for both ${ }^{13} \mathrm{CO}$ and ${ }^{12} \mathrm{CO}$. 

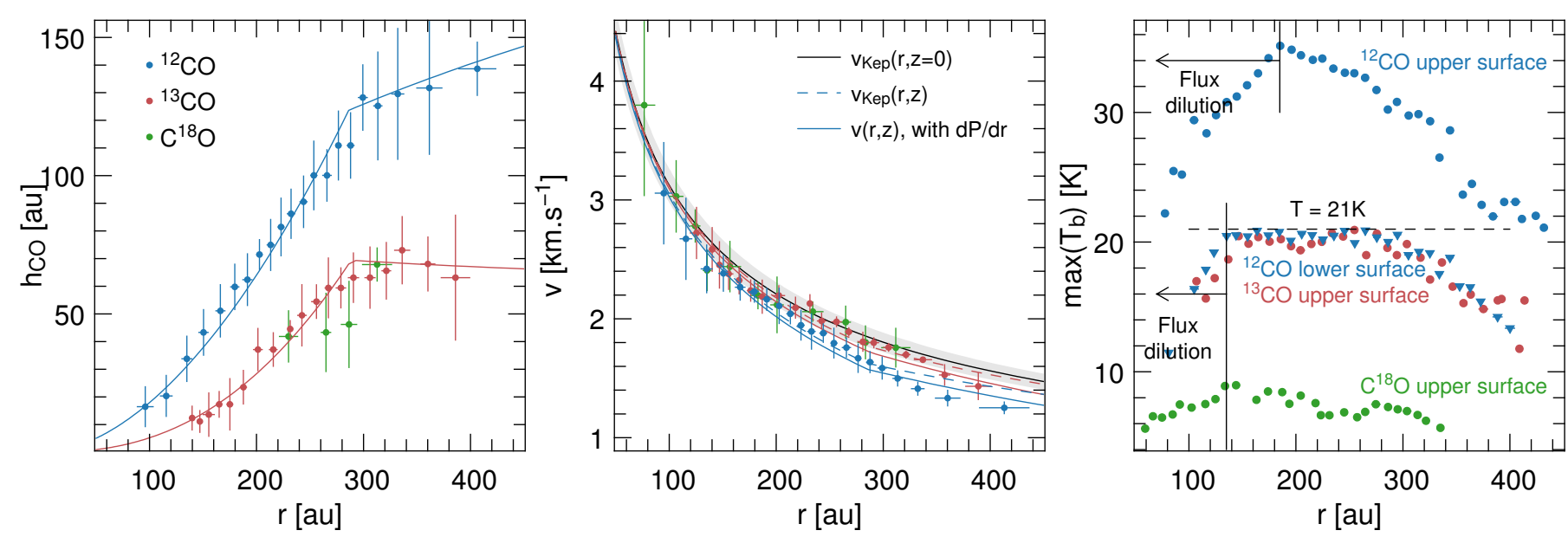

Fig. 4. Measured altitude, velocity, and brightness temperature of the $\mathrm{CO}$ layers as a function of radius: ${ }^{12} \mathrm{CO}$ (blue), ${ }^{13} \mathrm{CO}(\mathrm{red})$, and $\mathrm{C}^{18} \mathrm{O}(\mathrm{green})$. Data points were extracted for all channel maps and binned according to distance. The error bars represent the dispersion within the bins added quadratically to the uncertainty resulting from inclination $\left(48 \pm 3^{\circ}\right)$. Left: solid lines display a broken power-law fit (below and above $r=300$ au) for the ${ }^{12} \mathrm{CO}$ and ${ }^{13} \mathrm{CO}$. Centre: black solid line represents the Keplerian velocity for a $1 M_{\odot}$ central star, derived from the $\mathrm{C}^{18} \mathrm{O}$ position-velocity diagram (Appendix A). The shaded area represents an uncertainty of $0.1 M_{\odot}$. The red and blue dashed lines show the expected Keplerian velocities taking into account the altitude of the ${ }^{12} \mathrm{CO}$ and ${ }^{13} \mathrm{CO}$ layers. The red and blue solid lines display the velocity of the ${ }^{12} \mathrm{CO}$ and ${ }^{13} \mathrm{CO}$ layers taking into account the altitude and the pressure gradient term, as derived from our MCFOST model. Right: circles and triangles represent the temperature measured on the upper and lower surfaces, respectively. The brightness temperatures are similar on the upper and lower surfaces for ${ }^{13} \mathrm{CO}$ and $\mathrm{C}^{18} \mathrm{O}$; for clarity, only the upper surface is plotted.
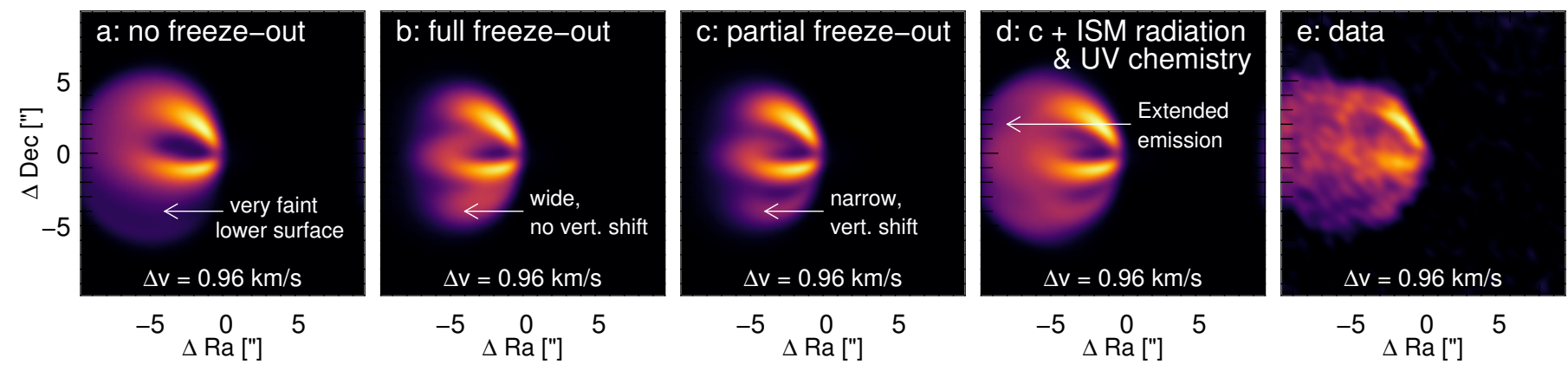

Fig. 5. Representative models of IM Lupi. The disc has been rotated so that the semi-major axis is horizontal. From left to right a): without any freeze-out of $\mathrm{CO} ; b$ ): with complete freeze-out of $\mathrm{CO}$, where $T<21 \mathrm{~K} ; c)$ : with depletion of gaseous $\mathrm{CO}$ by $10^{-4}$, where $T<21 \mathrm{~K}$; $d$ ): same as $c$ ) including the interstellar radiation field. The models are designed for qualitative comparison only. They are convolved to the ALMA resolution, but not degraded to the same noise level as the observations.

This is consistent with the expected pressure gradient as derived from the temperature and density structure of our MCFOST model (Fig. 4), which predicts a slightly smaller correction of $\approx 0.1 \mathrm{~km} \mathrm{~s}^{-1}$ at $400 \mathrm{au}$. This value depends on the local density and temperature gradients, which are not well constrained at these distances from the star, and the pressure gradient term might be able to fully explain the observed deviations. We note that the deviations from Keplerian velocities start at slightly different radii for the two isotopologues, $\approx 300$ au for ${ }^{12} \mathrm{CO}$ and $\approx 350$ au for ${ }^{13} \mathrm{CO}$. The difference is about one beam size and would need to be confirmed with higher spatial resolution observations. This behaviour is not captured by our model, suggesting that an additional process might be at play.

A possible explanation is that we could also be observing the base of a photo-evaporative wind. Haworth et al. (2017) showed that the large-scale halo of CO emission (>1000 au) described by Cleeves et al. (2016) could be interpreted as the result of a photoevaporative wind created by irradiation from the local (weak) external radiation field. The $\mathrm{CO}$ abundance in the flow remains high enough to be comparable to the observed large-scale emission around IM Lupi. Interestingly, as shown by Facchini et al. (2016) and Haworth et al. (2016), such a photo-evaporative wind can also result in a sub-Keplerian rotation field (see e.g. Fig. 10 of Haworth et al. 2016), between the outer edge of the disc and the $\mathrm{H}-\mathrm{H}_{2}$ transition. For the 1D models of IM Lupi presented by Haworth et al. (2017), the expected deviation from Keplerian rotation is at the $0.1-0.2 \mathrm{~km} \mathrm{~s}^{-1}$ level (Haworth, priv. comm.) and would also be consistent with our observations. Such a photo-evaporative wind could also explain why the deviations from Keplerian rotation seem to start closer in at high altitude where the density is lower, allowing the external radiation to penetrate further in. However, both Facchini et al. (2016) and Haworth et al. (2016) predict that the wind should also be associated with a radially increasing gas temperature profile. This is clearly not the case for IM Lupi, and more detailed modelling (e.g. 2D) might be necessary to test the hypothesis of external photo-evaporation further.

\subsection{Two-dimensional temperature structure and vertical CO snow line}

In the upper disc surface, we find that the ${ }^{12} \mathrm{CO}$ temperature is decreasing with radius, as expected from direct heating by the 


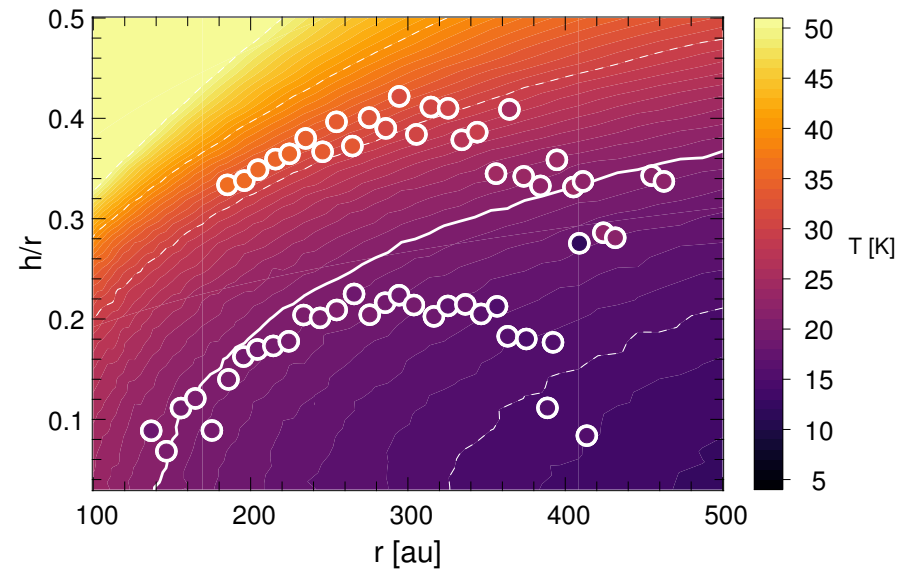

Fig. 6. Comparison of the measured temperature (points) with the temperature structure from our MCFOST model (background). The same colour map is used for the data and the model. The white contour is the $T=21 \mathrm{~K}$ level, and the dashed contours correspond to $T=15,30,40$, and $50 \mathrm{~K}$.

star (Fig. 4, right panel and Fig. 6). In the lower surface, on the other hand, the ${ }^{12} \mathrm{CO}$ temperature is virtually constant at $\approx 21 \mathrm{~K}$, between 130 and 300 au. Because we are looking at this surface through the disc, we are looking at the emitting CO layer closest to the midplane. At these large distances from the star, the dust continuum is optically thin (see Appendix C) and the measured $\mathrm{CO}$ brightness temperature is attenuated by at most $3 \%$ by continuum absorption. Because the whole CO layer is optically thick, we are observing its top in the upper surface, and its bottom in the lower surface. The difference in brightness temperature between the two sides of the disc is then a direct observation of the vertical temperature gradient.

The constant $21 \mathrm{~K}$ of the lower surface is consistent with the expected $\mathrm{CO}$ condensation temperature for binding onto pure CO ice (e.g. Sandford \& Allamandola 1988; Bisschop et al. 2006; Martín-Doménech et al. 2014; Fayolle et al. 2016). We interpret it as a strong indication that we are seeing gaseous $\mathrm{CO}$ just above the freeze-out region. This is reinforced by the measured brightness temperature of the ${ }^{13} \mathrm{CO}$ on the upper surface, which is extremely close to the ${ }^{12} \mathrm{CO}$ brightness temperature on the lower surface (Fig. 4, right panel). The combination of ${ }^{12} \mathrm{CO}$ and ${ }^{13} \mathrm{CO}$ allows us to probe both sides of the gaseous $\mathrm{CO}$ just above the freeze-out region. In other words, our method allows us to directly map the vertical snow line as a function of radius. The very similar temperature profiles, despite a typical factor $\approx 70$ in abundance, indicate that the lines remain optically thick and that we are indeed measuring the gas temperature in this region, hence directly measuring the $\mathrm{CO}$ condensation temperature.

A range of $\mathrm{CO}$ freeze-out temperatures have been estimated indirectly via thermo-chemical modelling and/or chemical imaging for the discs around the T Tauri star TW Hya (Qi et al. 2013: $17 \mathrm{~K}$; Zhang et al. 2017: $27 \mathrm{~K}$ ) and the Herbig Ae star HD 163296 (Qi et al. 2011: 19 K, Mathews et al. 2013: 19 K; Qi et al. 2015: 25 K), where freeze-out temperatures higher than $25 \mathrm{~K}$ would suggest ice mixtures where the binding energy is higher (Collings et al. 2003, 2004; Noble et al. 2012). The most meaningful comparison is probably with the results of Schwarz et al. (2016), who also measured directly the gas kinetic temperature from the peak brightness of the $\mathrm{CO}$ isotopologue lines of TW Hya. Interestingly, their measured freezeout temperature of $21 \mathrm{~K}$ is very close to the value we obtained for IM Lupi. In particular, the plateau observed in the kinetic temperature at $\approx 21 \mathrm{~K}$ (see their Fig. 3a) is strikingly similar to the one we observe for IM Lupi (Fig. 4, right panel). This suggests that the ice composition is likely similar, despite the significant age difference: 0.5-1 Myr for IMLupi (Mawet et al. 2012) compared to 2-10 Myr for TW Hya (Barrado y Navascués 2006; Vacca \& Sandell 2011).

The $\mathrm{C}^{18} \mathrm{O}$ emission has a brightness temperature of $\approx 8 \mathrm{~K}$ on both surfaces between 150 and $300 \mathrm{au}$. With an excitation temperature of $21 \mathrm{~K}$ as for ${ }^{13} \mathrm{CO}$, this means that the $\mathrm{C}^{18} \mathrm{O}$ optical depth is of the order of 0.5 . With a typical abundance ratio of $8: 1$, the corresponding ${ }^{13} \mathrm{CO}$ optical depth is $\approx 4$, confirming our assumption of $T_{\mathrm{ex}}=T_{\text {kin }}$. This moderate optical depth also explains why we observe the gas ${ }^{13} \mathrm{CO}$ in the dense region just above the $\mathrm{CO}$ snow line, but not at higher altitudes where it becomes optically thin (while ${ }^{12} \mathrm{CO}$ remains optically thick) due to the reduced densities.

At distances greater than $300 \mathrm{au}$, where the gas density drops, the temperature of the $\mathrm{CO}$ region above the snow line appears to decrease (as measured by both ${ }^{12} \mathrm{CO}$ and ${ }^{13} \mathrm{CO}$ ) with distance down to $\approx 15 \mathrm{~K}$ at $400 \mathrm{au}$. This is consistent with the expected dependence of the $\mathrm{CO}$ condensation temperature on the density (e.g. Collings et al. 2003; Bisschop et al. 2006; Hollenbach \& Gorti 2009). Detailed modelling by Cleeves et al. (2016) has shown that UV photo-desorption naturally explains the diffuse $\mathrm{CO}$ emission outside $400 \mathrm{au}$ (see also Fig. 5d). We might also observe this effect between 300 and 400 au, where the increasing external radiation field can allow the $\mathrm{CO}$ to remain in the gas phase at lower and lower temperatures as the density decreases with radius. Another possibility is that we are starting to see deviations from local thermodynamic equilibrium as the density becomes progressively lower than the critical density, and the measured excitation temperature can become lower than the actual kinetic temperature.

Closer in, the emission along the isovelocity curve becomes very compact and no longer fills the beam, resulting in a drop in the brightness temperature which can no longer be used to estimate the gas temperature. Interestingly, this drop does not occur at the same radius for the high altitude ${ }^{12} \mathrm{CO}$ emitting region $(r \approx 160 \mathrm{au})$ and for the CO layer just above the snow line $(r \approx 130 \mathrm{au})$. This is a direct indication that, in this range of radius, the vertical temperature gradient is becoming steeper at high altitude in the disc, as expected from models of irradiated discs (Fig. 6). When the beam becomes larger than the actual emitting region, a fraction of the beam is filled with emission originating from the wings of the local spectral line, i.e. with lower optical depth, meaning that part of the emission originates from further away along the line of sight. If the temperature gradient is shallow, the emission will remain at the same level, as seen for ${ }^{13} \mathrm{CO}, \mathrm{C}^{18} \mathrm{O}$, and ${ }^{12} \mathrm{CO}$ on the lower disc surface. This is consistent with the similar temperature we obtain while observing this layer from both sides with ${ }^{13} \mathrm{CO}$ and ${ }^{12} \mathrm{CO}$.

\section{Concluding remarks}

We have presented a general framework to measure directly the altitude, velocity, and temperature of the $\mathrm{CO}$ emitting layers in protoplanetary discs at intermediate inclination. These simple geometrical considerations were applied to IM Lupi. Because these measurements are performed directly on the ALMA channel maps and do not rely on any modeling of the disc, they put unbiased constraints on the disc structure, which in turn are critical to feed models of disc structure, dust evolution, and early stages of planet formation. 
The results are in agreement with the broad picture of how we understand protoplanetary discs, with a tapered-edge disc structure passively heated by the central star. The accuracy of our measurements is currently limited by the spatial resolution of $\approx 0.4^{\prime \prime}$ of our data. The presented framework is generic. We applied it to IM Lupi which holds an unusually large disc. However, the required high spectral resolution $\left(\lesssim 0.1 \mathrm{~km} \mathrm{~s}^{-1}\right)$ can be reached by ALMA down to spatial resolutions of $\approx 0.1-0.2^{\prime \prime}$ in a few hours to detect emission with $T_{\mathrm{b}} \approx 20 \mathrm{~K}$, making it possible to reproduce this analysis for a significant number of discs and constrain their thermal and kinematic structures in a consistent way.

At these high spatial resolutions, discs very often display structures, such as dust rings and gaps (ALMA Partnership et al. 2015; Isella et al. 2016; Fedele et al. 2017; van der Plas et al. 2017). The presented framework may offer a way to constrain local variations in altitude, temperature, and/or velocity of the CO layers in the vicinity of the observed structures, potentially pinpointing the mechanisms at the origin of these structures.

Acknowledgements. We thank T. J. Haworth, J. Lesur and C. McNally for the useful discussions. This paper makes use of the following ALMA data: ADS/JAO.ALMA\#2013.1.00798.S. ALMA is a partnership of ESO (representing its member states), NSF (USA), and NINS (Japan), together with NRC (Canada), NSC and ASIAA (Taiwan), and KASI (Republic of Korea), in cooperation with the Republic of Chile. The Joint ALMA Observatory is operated by ESO, AUI/NRAO, and NAOJ. The National Radio Astronomy Observatory is a facility of the National Science Foundation operated under cooperative agreement by Associated Universities, Inc. We acknowledge funding from ANR of France under contract number ANR-16-CE31-0013, from the Australian Research Council (ARC) under the Future Fellowship number FT170100040, from the National Centre for Competence in Research PlanetS supported by the Swiss National Science Foundation, and from the European Union Seventh Framework Programme FP7-2011 under grant agreement No. 284405.

\section{References}

Aikawa, Y. 2007, ApJ, 656, L93

Aikawa, Y., Furuya, K., Nomura, H., \& Qi, C. 2015, ApJ, 807, 120

ALMA Partnership, Brogan, C. L., Pérez, L. M., et al. 2015, ApJ, 808, L3

Barrado y Navascués, D. 2006, A\&, 459, 511

Barrière-Fouchet, L., Gonzalez, J.-F., Murray, J. R., Humble, R. J., \& Maddison,

S. T. 2005, A\&A, 443, 185

Bisschop, S. E., Fraser, H. J., Öberg, K. I., van Dishoeck, E. F., \& Schlemmer, S. 2006, A\&A, 449, 1297

Blum, J., \& Wurm, G. 2008, ARA\&A, 46, 21

Boehler, Y., Weaver, E., Isella, A., et al. 2017, ApJ, 840, 60

Brauer, F., Dullemond, C. P., \& Henning, T. 2008, A\&A, 480, 859

Ciesla, F. J., \& Cuzzi, J. N. 2006, Icarus, 181, 178

Cleeves, L. I., Öberg, K. I., Wilner, D. J., et al. 2016, ApJ, 832, 110

Collings, M. P., Dever, J. W., Fraser, H. J., McCoustra, M. R. S., \& Williams,

D. A. 2003, ApJ, 583, 1058

Collings, M. P., Anderson, M. A., Chen, R., et al. 2004, MNRAS, 354, 1133

Dartois, E., Dutrey, A., \& Guilloteau, S. 2003, A\&A, 399, 773 de Gregorio-Monsalvo, I., Ménard, F., Dent, W., et al. 2013, A\&A, 557, A133 Dominik, C., Blum, J., Cuzzi, J. N., \& Wurm, G. 2007, in Protostars and Planets V, eds. B. Reipurth, D. Jewitt, \& K. Keil, 783

Dorschner, J., Begemann, B., Henning, T., Jaeger, C., \& Mutschke, H. 1995, A\&A, 300, 503

Dutrey, A., Guilloteau, S., Piétu, V., et al. 2017, A\&A, 607, A130

Facchini, S., Clarke, C. J., \& Bisbas, T. G. 2016, MNRAS, 457, 3593

Fayolle, E. C., Balfe, J., Loomis, R., et al. 2016, ApJ, 816, L28

Fedele, D., Carney, M., Hogerheijde, M. R., et al. 2017, A\&A, 600, A72

Flaherty, K. M., Hughes, A. M., Rosenfeld, K. A., et al. 2015, ApJ, 813, 99

Fromang, S., \& Nelson, R. P. 2009, A\&A, 496, 597

Furuya, K., \& Aikawa, Y. 2014, ApJ, 790, 97

Gaia Collaboration (Brown, A. G. A., et al.) 2016, A\&A, 595, A2

Ginski, C., Stolker, T., Pinilla, P., et al. 2016, A\&A, 595, A112

Haworth, T. J., Boubert, D., Facchini, S., Bisbas, T. G., \& Clarke, C. J. 2016, MNRAS, 463, 3616

Haworth, T. J., Facchini, S., Clarke, C. J., \& Cleeves, L. I. 2017, MNRAS, 468, L108

Hollenbach, D., \& Gorti, U. 2009, ApJ, 703, 1203

Huang, J., Öberg, K. I., Qi, C., et al. 2017, ApJ, 835, 231

Isella, A., Guidi, G., Testi, L., et al. 2016, Phys. Rev. Lett., 117, 251101

Lagage, P.-O., Doucet, C., Pantin, E., et al. 2006, Science, 314, 621

Laibe, G., Gonzalez, J.-F., \& Maddison, S. T. 2012, A\&A, 537, A61

Martín-Doménech, R., Muñoz Caro, G. M., Bueno, J., \& Goesmann, F. 2014, A\&A, 564, A8

Mathews, G. S., Klaassen, P. D., Juhász, A., et al. 2013, A\&A, 557, A132

Mawet, D., Absil, O., Montagnier, G., et al. 2012, A\&A, 544, A131

McMullin, J. P., Waters, B., Schiebel, D., Young, W., \& Golap, K. 2007, in Astronomical Data Analysis Software and Systems XVI, eds. R. A. Shaw, F. Hill, \& D. J. Bell, ASP Conf. Ser., 376, 127

Noble, J. A., Theule, P., Mispelaer, F., et al. 2012, A\&A, 543, A5

Öberg, K. I., Furuya, K., Loomis, R., et al. 2015, ApJ, 810, 112

Panić, O., Hogerheijde, M. R., Wilner, D., \& Qi, C. 2009, A\&A, 501, 269

Pavlyuchenkov, Y., Semenov, D., Henning, T., et al. 2007, ApJ, 669, 1262

Pinilla, P., Birnstiel, T., Ricci, L., et al. 2012, A\&A, 538, A114

Pinte, C., \& Laibe, G. 2014, A\&A, 565, A129

Pinte, C., Ménard, F., Duchêne, G., \& Bastien, P. 2006, A\&A, 459, 797

Pinte, C., Ménard, F., Berger, J. P., Benisty, M., \& Malbet, F. 2008, ApJ, 673, L63

Pinte, C., Harries, T. J., Min, M., et al. 2009, A\&A, 498, 967

Pinte, C., Dent, W. R. F., Ménard, F., et al. 2016, ApJ, 816, 25

Qi, C., D'Alessio, P., Öberg, K. I., et al. 2011, ApJ, 740, 84

Qi, C., Öberg, K. I., Wilner, D. J., et al. 2013, Science, 341, 630

Qi, C., Öberg, K. I., Andrews, S. M., et al. 2015, ApJ, 813, 128

Ros, K., \& Johansen, A. 2013, A\&A, 552, A137

Rosenfeld, K. A., Andrews, S. M., Wilner, D. J., Kastner, J. H., \& McClure, M. K. 2013, ApJ, 775, 136

Sandford, S. A., \& Allamandola, L. J. 1988, Icarus, 76, 201

Schwarz, K. R., Bergin, E. A., Cleeves, L. I., et al. 2016, ApJ, 823, 91

Shakura, N. I., \& Sunyaev, R. A. 1973, A\&A, 24, 337

Testi, L., Birnstiel, T., Ricci, L., et al. 2014, Protostars and Planets VI, 339

Vacca, W. D., \& Sandell, G. 2011, ApJ, 732, 8

van der Plas, G., Wright, C. M., Ménard, F., et al. 2017, A\&A, 597, A32

van't Hoff, M. L. R., Walsh, C., Kama, M., Facchini, S., \& van Dishoeck, E. F. 2017, A\&A, 599, A101

Weidenschilling, S. J. 1977, MNRAS, 180, 57

Woitke, P., Kamp, I., \& Thi, W. 2009, A\&A, 501, 383

Woitke, P., Min, M., Pinte, C., et al. 2016, A\&A, 586, A103

Zhang, K., Bergin, E. A., Blake, G. A., Cleeves, L. I., \& Schwarz, K. R. 2017,

Nat. Astron., 1, 0130 

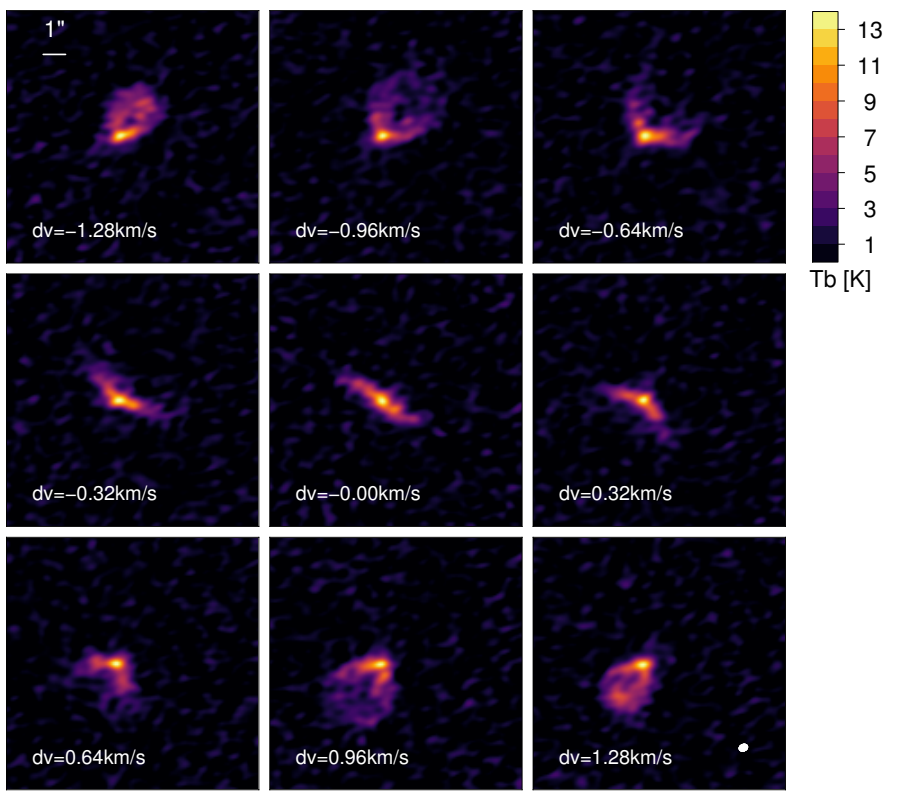

Fig. A.1. $\mathrm{C}^{18} \mathrm{O}$ channel map, with the same channel width of $80 \mathrm{~m} / \mathrm{s}$ as in Fig. 1.

\section{Appendix $A: C^{18} O$ observational results}

The $\mathrm{C}^{18} \mathrm{O}$ channel maps are presented in Fig. A.1. In Fig. B.1, we compare positions-velocity diagrams along the disc major axis with curves representing Keplerian rotation in a geometrically thin disc with an inclination of $48^{\circ}$. The position-velocity diagrams are nicely reproduced by a central mass of $1 \pm 0.1 M_{\odot}$, assuming a distance of $161 \mathrm{pc}$. This is in agreement with the value used by Panić et al. (2009; rescaling for the difference in distance) and Cleeves et al. (2016).

\section{Appendix B: Model description and parameters}

We use the Monte Carlo radiative transfer code MCFOST (Pinte et al. 2006, 2009) to build a simple model of the CO emission. Our model is an extension of the model presented in Pinte et al. (2008), where the radial power-law surface density was replaced by a tapered-edge structure, following Panić et al. (2009). This model is only meant to provide a qualitative description of the ALMA band 6 data. A complete fit of all the observations of IM Lupi is beyond the scope of this paper, and we refer the readers to the work by Cleeves et al. (2016) for the most recent global model of IM Lupi.

The central object is described by a photosphere at $3900 \mathrm{~K}$, a radius of $2.15 R_{\odot}$ and a mass of $1 M_{\odot}$. An ultra-violet excess is added to the phosphere, defined by a parameter $f_{\mathrm{UV}}$, which describes the fractional luminosity excess between 91.2 and $250 \mathrm{~nm}$.

An external, isotropic, interstellar radiation field is added in model $d$ presented in Fig. 5. It is composed of a highly diluted UV field, an infrared background radiation field, and the cosmic microwave background. We follow the same prescription as Woitke et al. (2016, see their Appendix A.4 for details). In this work, the UV component is scaled to $4 G_{\circ}$, following the results of Cleeves et al. (2016), who estimated the local radiation field from geometrical arguments based on HIPPARCos data.

We consider an axisymmetric flared density structure with a Gaussian vertical profile. The disc scale height is described by a power-law

$h(r)=h_{0}\left(r / r_{0}\right)^{\beta}$

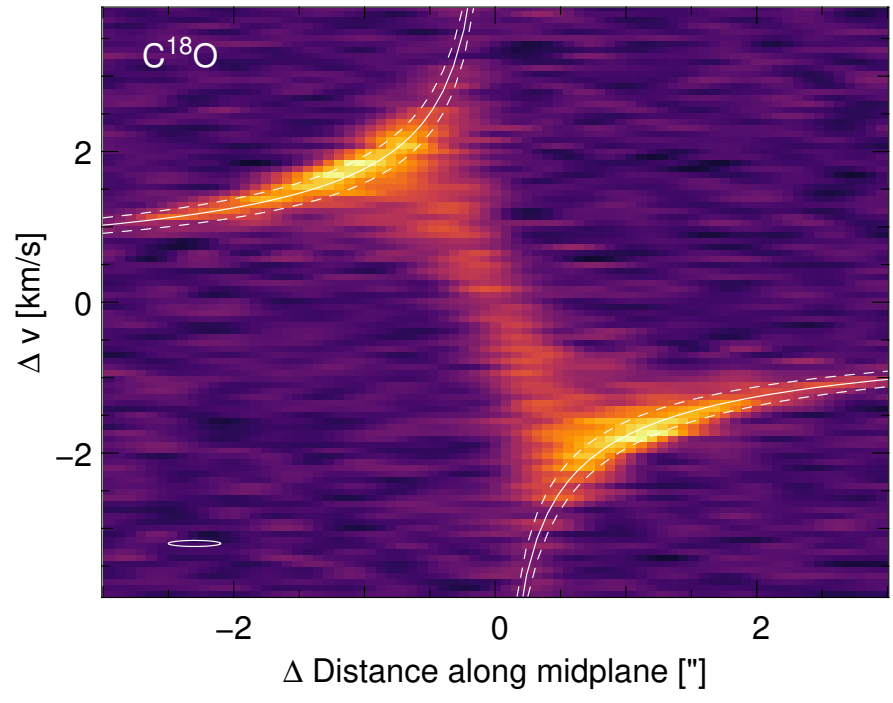

Fig. B.1. Position-velocity diagram of the continuum subtracted $\mathrm{C}^{18} \mathrm{O}$ line along the disc major axis. The $\Delta \mathrm{v}$ is relative to the systemic velocity of $4.5 \mathrm{~km} \mathrm{~s}^{-1}$. The solid line shows the midplane Keplerian velocity for a stellar mass of $1 M_{\odot}$ and an inclination of $48^{\circ}$. The dashed lines correspond to stellar masses of 0.9 and $1.1 M_{\odot}$. The data have been spectrally smoothed to a resolution of $80 \mathrm{~m} \mathrm{~s}^{-1}$. The spectro-spatial resolution is indicated in the lower left corner.

and its surface density by

$\Sigma(r) \propto r^{-\gamma} \exp \left(-\left(r / r_{\text {tap }}\right)^{2-\gamma_{\exp }}\right)$,

where $r$ is the radial coordinate in the equatorial plane and $h_{0}$ is the scale height at the radius $r_{0}=100 \mathrm{au}$. The disc extends from $r_{\text {in }}$, that we fixed to $0.3 \mathrm{au}$, to an outer $r_{\text {out }}$, set to 8 times the critical radius $r_{\text {tap }}$, i.e. where the density is so low that the actual value does not affect any of the synthetic observables.

Dust grains are defined as homogeneous and spherical particles with sizes distributed according to the power law $\mathrm{d} n(a) \propto$ $a^{-3.5} \mathrm{~d} a$ between $a_{\min }=0.03 \mu \mathrm{m}$ and $a_{\max }$. Optical properties are computed using the Mie theory, adopting the same olivine stoichiometry from Dorschner et al. (1995) as in Pinte et al. (2008).

Vertical dust settling is implemented by following the same prescription as in Pinte et al. (2016) and Fromang \& Nelson (2009, see their Eq. (19)), where the degree of dust settling is set by varying the turbulent viscosity coefficient $\alpha_{\mathrm{SS}}$ (Shakura \& Sunyaev 1973).

For the dust radial migration, we follow the simple prescription introduced in Pinte \& Laibe (2014). The maximum expected grain size is set to the optimal size of migration

$s_{\mathrm{opt}}(r)=\frac{\rho(r, z=0) c_{\mathrm{s}}(r, z=0)}{\rho_{\mathrm{dust}} \Omega_{\mathrm{K}}}$,

where $\rho_{\mathrm{g}}$ and $c_{\mathrm{S}}$ are the gas density and sound speed, $\rho_{\text {dust }}$ the intrinsic dust density, and $\Omega_{\mathrm{K}}$ the Keplerian frequency respectively.

$\mathrm{CO}$ chemistry is dominated by a few key mechanisms: photodissociation by UV radiation in the upper layers, condensation on the dust grains at low temperatures $(T \lesssim 20 \mathrm{~K})$, and photodesorption of the $\mathrm{CO}$ ice by UV radiation in the outer disc. We have implemented a very simple model of the CO chemistry, which was calibrated from ProDiMo models (Woitke et al. 2016, see e.g. their Fig. C.2). We initially assume that the $\mathrm{CO}$ abundance $X(\mathrm{CO})$ is constant everywhere. $\mathrm{CO}$ is photo-dissociated, i.e. we set its gas abundance to 0 if

$\log (\chi / n)>-6$, 
Table B.1. Parameters of our disc model, as derived by our genetic algorithm from fitting of the Band 6 ALMA data only.

\begin{tabular}{lc}
\hline \hline Model parameter & Value \\
\hline$a_{\max }[\mu \mathrm{m}]$ & 2300 \\
$M_{\text {dust }}\left[M_{\odot}\right]$ & $1.75 \times 10^{-3}$ \\
gas/dust radio & 347 \\
$\alpha_{\text {SS }}$ (dust settling) & $9 \times 10^{-3}$ \\
$\beta$ & 1.19 \\
$\gamma$ & 0.48 \\
$\gamma_{\text {exp }}[\mathrm{au}]$ & 0.32 \\
$R_{\text {tap }}[\mathrm{au}$ & 284 \\
$h$ at $100 \mathrm{au}[\mathrm{au}]$ & 12.9 \\
$v_{\text {turb }}\left[\mathrm{km} \mathrm{s}{ }^{-1}\right]$ & 0.08 \\
$X\left({ }^{12} \mathrm{CO}\right)$ & $5 \times 10^{-5}$ \\
$X\left({ }^{13} \mathrm{CO}\right)$ & $7 \times 10^{-7}$ \\
$X\left(\mathrm{C}{ }^{18} \mathrm{O}\right)$ & $1 \times 10^{-7}$ \\
$\epsilon$ & $8 \times 10^{-5}$ \\
$f_{\mathrm{UV}}$ & 0.09 \\
\hline
\end{tabular}

where $n$ is the number density of hydrogen atoms and $\chi$ describes the intensity of the UV radiation field, following Woitke et al. (2009)

$\chi=\frac{1}{F^{\text {Draine }}} \int_{91.2 \mathrm{~nm}}^{205 \mathrm{~nm}} u_{\lambda} \mathrm{d} \lambda$,

with $F^{\text {Draine }}=1.921 \times 10^{12} \mathrm{~m}^{-2} \mathrm{~s}^{-1}$, and where $u_{\lambda}$ is the energy density of the radiation field which was computed by MCFOST at each point in the model.

$\mathrm{CO}$ is frozen out on the dust grains if $T<21 \mathrm{~K}$, i.e. its abundance is multiplied by a factor $\epsilon<1$, except if

$\log (\chi / n)>-7$,

where $\mathrm{CO}$ is photo-desorbed.

The dust temperature structure is computed using MCFOST. We then assume that the gas temperature is equal to the dust temperature and that the $\mathrm{CO}$ emission is at local thermodynamic equilibrium, and compute the synthetic channel map via a raytracing method.

As our aim was simply to build an illustrative model, we only adjusted the Band $6 \mathrm{CO}$ data in the image place via a genetic algorithm (each generation was composed of 100 models and the genetic algorithm was run for 50 generations). As a consequence, our model may not be a unique solution, and the model parameters presented in Table B.1 must be interpreted with care.

\section{Appendix C: Continuum optical depth}

To assess the continuum optical depth of the disc, a more accurate radiative transfer model of the continuum emission (Fig. C.1) is required. We use the same iterative procedure as in Pinte et al. (2016) for HL Tau. In short, we first extract a brightness profile along the disc major axis in the CLEANed map (average of both sides). We deconvolve this profile by a Gaussian corresponding to the size of the beam in that direction. We convert the deconvolved brightness profile into a surface density profile assuming an initial power-law radial temperature profile (exponent $=-0.5$ ), from which we compute a MCFOST model.

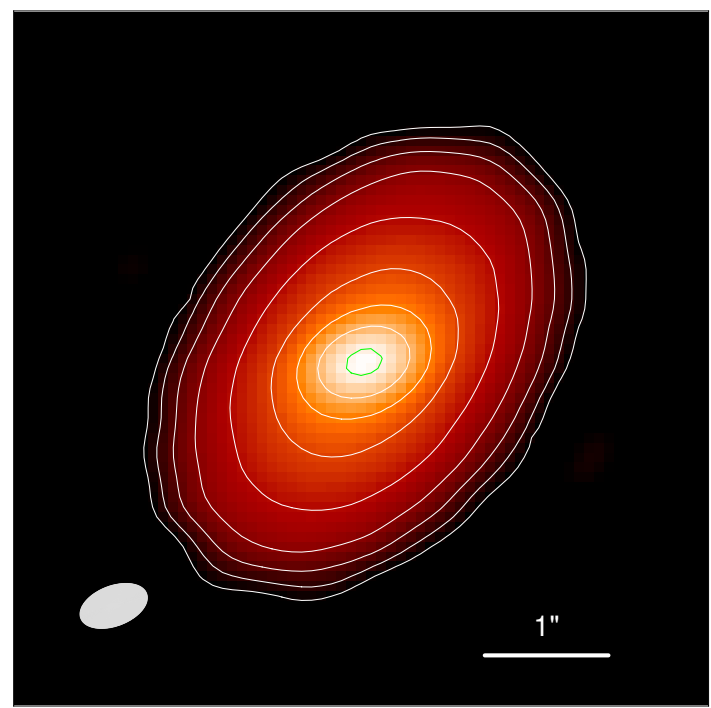

Fig. C.1. Continuum image of IM Lupi at $\lambda=1.3 \mathrm{~mm}$. Contours begin at $4 \sigma$ and step in factors of 2 in intensity up to $1024 \sigma$ (green contour), where $\sigma=0.05 \mathrm{mJy} / \mathrm{beam}$ is the RMS measured on the map away from the source.

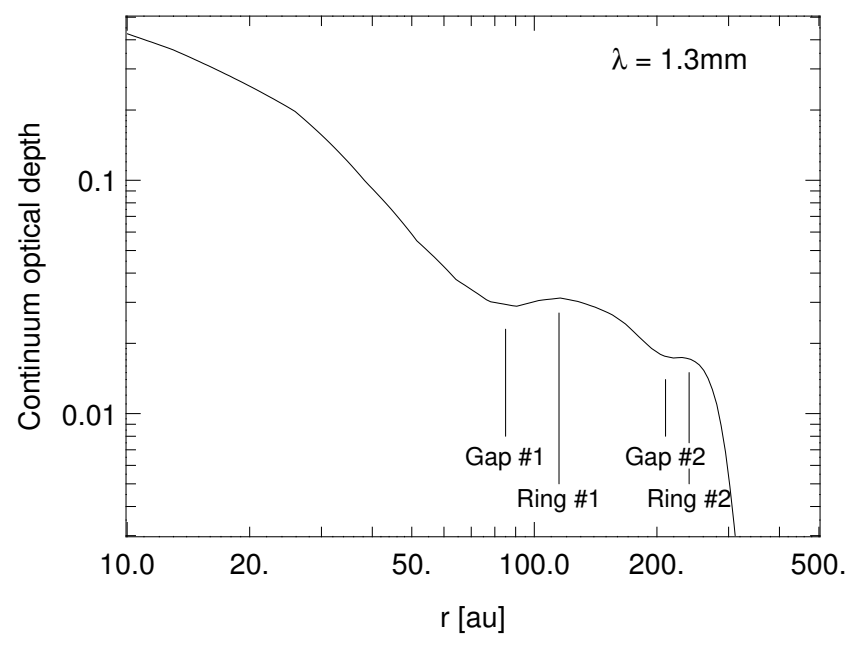

Fig. C.2. Continuum optical depth along the line of sight at $\lambda=1.3 \mathrm{~mm}$ as a function of radius. The optical depth takes the inclination of the disc into account and is measured along the disc major axis.

We compare the resulting synthetic surface brightness profile with the observed one and iteratively correct the surface density until the synthetic brightness profile does not change by more than $1 \%$ at any radius. We reach convergence after about ten iterations. The resulting continuum optical depth as a function of radius is presented in Fig. C.2.

In the regions we are studying in this paper, i.e. outside $100 \mathrm{au}$, the maximum optical depth is $\approx 0.03$, meaning that the flux on the lower surface of the disc is attenuated by at most $3 \%$, due to continuum absorption by the dust.

Interestingly, the optical depth and corresponding dust surface density show two dips at $\approx 85$ and $210 \mathrm{au}$, suggesting the presence of two gaps, and the corresponding two rings peaking at $\approx 115$ and $240 \mathrm{au}$. They very likely correspond to the rings observed by Cleeves et al. (2016) at $\approx 150$ and 250 au, after applying an unsharp mask. 